\title{
CULTIVOS Y CRECIMIENTO \\ DE LA POBLACIÓN ESCLAVA: \\ EL CASO DE GUYANA EN EL SIGLO XIX
}

\author{
CARLOS NEWLAND \\ Universidad Torcuato Di Tella \\ y EMILIO PARRADO \\ Universidad de Chicago
}

\section{INTRODUCCIÓN}

Uno de los temas más discutidos en la historia demografica ha sido el crecimiento de las poblaciones esclavas del continente americano. En particular ha llamado la atención la diversa experiencia de las distintas regiones y paises. Mientras que en Estados Unidos durante la primera mitad del siglo xIx la población esclava creció a una tasa superior al $2 \%$, en el Caribe sufrió una dis. minución constante, disminución que variaba de isla en isla, pero que en general alcanzaba un $3 \%$ anual '. En el Brasil, única nación de América del Sur con una gran concentración de mano de obra forzada, la situación fue interme. dia, con una caída anual de algo más de $1 \%^{2}$. Tanto en el Caribe como en Brasil se buscó compensar la pérdida natural de población mediante la importación de africanos, actividad que se dificultó luego de la prohibición británica de la trata en $1807^{3}$.

Se han presentado múltiples teorias para explicar el diverso comportamiento demográfico de los esclavos. Una explicación se ha concentrado en las prácticas de lactancia y su conocido efecto negativo sobre la natalidad. En los Estados Unidos existiría un periodo reducido de amamantamiento, mientras que en el Caribe y en Brasil seria más extenso, debido que se mantuvieron vi-

' Sobre el crecimiento de la población esclava en Estados Unidos y Jamaica, vease Engerman (1976), p. 271 . Una estimación para una parte de la población esclava de Cuba señala un crecimiento anual negativo de $1,1 \%$. Vease Bergad (1990), p. 100.

2 Merrick y Graham (1979), pp. 61, 65; Schwartz (1985), p. 368.

' La prohibición efectiva de la trata en Brasil sólo se hizo efectiva en 1851. 
vas costumbres africanas por los inmigrantes africanos 4 . Una herencia cultural adicional de África presente en el Caribe y Brasil seria la de mantener un periodo largo de abstinencia sexual después del parto, lo que impediria a la mujer quedar inmediatamente embarazada 5 . Tambien se ha argumentado que en el Caribe los esclavos accedieron a una dieta mucho más pobre en proteinas que en Estados Unidos, lo que perjudicó su salud y aumentó la mortalidad infantil ${ }^{\circ}$. Finalmente, una explicación usual es que en el Caribe y en Brasil -a diferencia de Estados Unidos- los sexos estaban muy desbalanceados por la adquisición predominante de varones; sencillamente no existian suficientes mujeres reproductoras para mantener la población '.

En este trabajo se analizan los determinantes de crecimiento de la población esclava, poniendo especial énfasis en el cultivo del algodón, café y azúcar, tanto sobre la natalidad, como sobre la mortalidad. Para analizar el impacto demográfico de los distintos cultivos es preferible concentrarse en un solo ám. bito geográfico, para asi evitar el influjo de distintos entornos económicos o institucionales. A tal efecto se ha utilizado un conjunto de datos de la colonia británica de Guyana en la primera mitad del siglo XIX, que presenta la ventaja de incluir información demográfica sobre plantaciones de los diversos cultivos mencionados. El análisis de la muestra permite contrastar algunas de las teorías explicativas del cambio de población, como comparar sus conclusiones con los obtenidos en otros estudios.

\section{LA MUESTRA}

Entre los registros de esclavos generados por solicitud del Parlamento británico, con motivo del debate sobre su emancipación, se encuentra un relevamiento sobre el comportamiento demográfico en una serie de plantaciones de Demerara y Essequibo, dos distritos de Guyana ${ }^{8}$. Los registros proveen información sobre el número de nacimientos y defunciones para distintos tipos de plantaciones durante los tres años transcurridos entre mayo de 1829 y mayo

+ Véase Klein y Engerman (1978), y en su apoyo, con evidencia histórica bioantropológica, Handler y Corruccini (1986); la aplicacion al Brasil, en Schwartz (1985), pp. 363-64.

S Véase Higman (1976), p. 119

- Para Brasil, véase Schwartz (1985), p. 364.

Véase Kiple (1984), pp. 117-118.

8 "Summary of the Return of the Slave Population, distinguishing the variofls Plantations, and the actual Amount of Unattached or Personal Slaves, in each Parish of the District of Deme. rary and Essequebo, of the Colony of British Guiana, for the 31st May 1832», Parliamentary Papers (1833), XXVI, pp. 4.11. 
de 1832, el lapso cubierto por el relevamiento. Por las caracteristicas de la información, los registros permiten estudiar la evolución del numero de esclavos durante el periodo y evaluar el impacto de los diferentes tipos de cultivos sobre las tasas de natalidad, mortalidad y crecimiento natural.

El registro cubrió algo más de la mitad de la población de la colonia británica, cuyos esclavos obtuvieron su libertad en 1834. Las características de la muestra se presentan en el Cuadro $A$; debe señalarse que se ha incluido en la misma únicamente las plantaciones de café, algodón y azúcar, dejando de lado los establecimientos mixtos o los ocupados en otros cultivos ${ }^{9}$. Como ocurría en general en el Caribe, de las 194 plantaciones analizadas la mayor parte $(78 \%)$ estaba ocupada en el cultivo de azúcar, y sólo el $15 \%$ y el $7 \%$ estaban dedicadas, respectivamente, al cultivo de café y algodón. En general la población de los dos sexos era equilibrada, con una pequeña preponderancia masculina en todos los tipos de plantación. Las fuertes diferencias entre la cantidad de varones y mujeres que se encontraban a principios del siglo xix se habian erosionado considerablemente debido a la prohibición de la trata ${ }^{10}$. Al inicio del periodo en 1829, el total de la población esclava en la muestra ascendia a 44.309. Hacia 1832 la población habla disminuido a 42.190 , lo que significó una reducción de $4,5 \%$ y una tasa anual de crecimiento negativa de $1,7 \%$. Sin embargo, el comportamiento demográfico no es homogeneo para los distintos tipos de plantaciones. El Cuadro A muestra que mientras que la tasa de crecimiento natural en las plantaciones de azúcar y café era negativa, con niveles de -17.5 y $-14,1$ por mil, en las plantaciones de algodón la tasa de crecimiento vegetativo era positiva, alcanzando el 3,1 por mil. El análisis por cultivo resalta el efecto negativo sobre la mortalidad y natalidad tanto del azúcar como del café, conjunto de plantaciones en que la población estaba decreciendo. El algodón muestra una realidad muy distinta con respecto a los otros tipos de cultivo, puesto que en este caso la población creció -mínimamentetanto por una mayor natalidad como por una menor mortalidad.

Para una mejor comprensión del comportamiento demográfico en las distintas plantaciones y para analizar la relevancia estadística de las variaciones observadas se efectuó un análisis de regresión multivariado, donde no sólo se investigó el efecto de los cultivos sobre natalidad y mortalidad en cada plantación, sino también del desequilibrio entre sexos y de la posible contribución de la mortalidad infantil a la baja tasa de natalidad.

\footnotetext{
Tambien se dejaron de lado unas pocas plantaciones de diez o menos esclavos. Además, dado que la unidad de analisis es la plantacion, los esclavos no pertenecientes a una de ellas debieron descartarse.

10 Sobre la evolución del desequilibrio sexual en Demerara entre 1817 y 1830 , véase Hig. $\operatorname{man}(1976)$, p. 137.
} 
CUADRO A

Indicadores demográficos medios por tipo de cultivo

\begin{tabular}{|c|c|c|c|}
\hline & Azúcar & Café & Algodón \\
\hline Población promedio en 1829 & 259 & 101 & 156 \\
\hline Población promedio en 1832 . & 246 & 96 & 158 \\
\hline Numero de plantaciones ............. & 152 & 29 & 13 \\
\hline Tasa varón mujer $\ldots \ldots \ldots \ldots \ldots \ldots$ & 1,21 & 1,26 & 1,15 \\
\hline Tasa bruta de natalidad $(\times 1.000)$ & 18,71 & 17,14 & 27,09 \\
\hline Tasa bruta de mortalidad $(\times 1.000\}$ & 36,24 & 31.26 & 23,99 \\
\hline 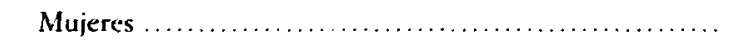 & 26,00 & 26,11 & 18,83 \\
\hline Varones $\ldots \ldots \ldots \ldots \ldots \ldots$ & 41,00 & 33,9 & 26,89 \\
\hline Tasa de crecimiento natural ......... & $-17,53$ & $-14,11$ & 3,10 \\
\hline
\end{tabular}

\section{LOS DETERMINANTES DE LA MORTALIDAD}

El Cuadro B presenta el resultado de los modelos de regresión estimados. Como las variables dependientes son tasas, $y$ a fin de evitar influyentes efectos provenientes de plantaciones con reducido número de esclavos, los modelos estimados fueron ponderados por la población en cada plantación.

La primera columna del Cuadro B muestra las resultados de la estimación del modelo que predice la tasa de mortalidad total en las distintas plantaciones. Se observa una mortalidad menor en las plantaciones de algodón con respecto a las de café y azúcar; el café presenta un valor intermedio. La diferencia entre los cultivos es más reveladora si se compara la mortalidad por sexo. En el Cuadro A se nota que los varones en general presentan una mayor mortalidad que las mujeres, un resultado coincidente con el obtenido para otras investigaciones del Caribe ". Según la segunda y tercera columnas del Cuadro B, los varones se ven más afectados por el cultivo en que trabajaban que las mujeres ${ }^{12}$. La producción de azúcar (y en menor medida de café) perjudicaba sobre todo la salud del hombre. Ello pudo deberse a que las tareas más duras $o$ arriesgadas estuvieron reservadas a los varones o a la mayor fortaleza biológi-

$"$ Sobre el mismo resultado obrenido para los esclavos de Trinidad, véase John (1988), p. 109; para Jamaica, Dunn (1990), pp. 51.52. Sobre el tema. véase tambien Kiple (1981), pp. 149.150.

12 Para Trinidad un estudio no ha encontrado una diferencia en la mortalidad femenina entre el cafe, azúcar y algodón. Véase John (1988), p. 117. 
ca de las mujeres. En el caso del azúcar, donde se encuentra la mayor mortalidad, la salud del hombre se vio afectada por jornadas laborables extremadamente largas e intensas ${ }^{13}$, por las posibilidades de lastimarse cortando caña y en el molino, por el efecto del calor en el cuarto de calderas y por las quemaduras sufridas por el jugo en ebullición ${ }^{14}$. En el caso de la mortalidad femenina, se observa que la variable que expresa la razón entre hombres y mujeres tiene un efecto positivo significativo: a medida que este indicador se incrementa, o sea, hay más hombres por mujer, la tasa de mortalidad femenina tiende a declinar. Una explicación posible es que existiese una mayor protección o cuidado de la mujer donde ésta fuera más escasa.

\section{CUADRO B}

Resultados del análisis de regresión (ponderado por población)

\begin{tabular}{|c|c|c|c|c|c|}
\hline & \multicolumn{5}{|c|}{ Variables dependientes } \\
\hline & \multicolumn{3}{|c|}{ Tasa bruta de mortalidad } & \multirow{2}{*}{\multicolumn{2}{|c|}{ Tasa bruta de natalidad }} \\
\hline & Total & Mujeres & Varones & & \\
\hline Café & $\begin{array}{c}-5,08 \\
(3,30)\end{array}$ & $\begin{array}{r}0,63 \\
(3,35)\end{array}$ & $\begin{array}{l}-7,21 * \\
(4,05)\end{array}$ & $\begin{array}{l}-1,39 \\
(2,06)\end{array}$ & $\begin{array}{l}-2,42 \\
(1,96)\end{array}$ \\
\hline Algodón & $\begin{array}{l}-12,13 * \\
(3,87)\end{array}$ & $\begin{array}{l}-7,75 * \\
(3,92)\end{array}$ & $\begin{array}{c}-13,97 * \\
(4,74)\end{array}$ & $\begin{array}{c}8,2 * \\
(2,41)\end{array}$ & $\begin{array}{r}5,73 * \\
(2,34)\end{array}$ \\
\hline Razón hombre/mujer ..... & $\begin{array}{l}1.981 \\
(2,35)\end{array}$ & $\begin{array}{c}-10,16: \\
(2,38)\end{array}$ & $\begin{array}{r}2,44 \\
(2,87)\end{array}$ & $\begin{array}{l}-3,29 * \\
(1,46)\end{array}$ & $\begin{array}{l}-2,89 * \\
(1,39)\end{array}$ \\
\hline Tasa bruta de mortalidad .. & & & & & $\begin{array}{l}-0,203 \\
(0,043)\end{array}$ \\
\hline Constante & $\begin{array}{l}33,84 * \\
(2,97)\end{array}$ & $\begin{array}{l}38,28 * \\
(3,01)\end{array}$ & $\begin{array}{l}38,04= \\
(3,63)\end{array}$ & $\begin{array}{l}22,68 \\
(1,85)\end{array}$ & $\begin{array}{l}29,56 " \\
(2,28)\end{array}$ \\
\hline$F \ldots \ldots \ldots \ldots \ldots \ldots \ldots$ & $4,17^{*}$ & $7,21 *$ & 4,03 * & $6,03 *$ & $10,64^{*}$ \\
\hline $\begin{array}{l}\text { R.cuad. aj. } \ldots \ldots \ldots \ldots \ldots \\
\mathrm{N} \cdot{ }^{\circ} \ldots \ldots \ldots \ldots \ldots \ldots\end{array}$ & $\begin{array}{l}0,047 \\
194\end{array}$ & $\begin{array}{l}0,088 \\
194\end{array}$ & $\begin{array}{l}0,045 \\
194\end{array}$ & $\begin{array}{l}0,072 \\
194\end{array}$ & $\begin{array}{l}0,167 \\
194\end{array}$ \\
\hline
\end{tabular}

* $p<0,05$.

NotA: Error estandar entre paréntesis.

"Higman (1984), p. 188.

it John (1988), p. 106. En T'rinidad el azúcar también producia una mayor mortalidad que los otros cultivos, especialmente respecto del algodón. Véase John (1988), p. 116. 
Los resultados de las estimaciones sugieren la existencia de tres regimenes de mortalidad: el algodón, con baja tasa de mortalidad; el café, con una tasa de mortalidad intermedia, y el azúcar, con el más alto nivel de mortalidad. El efecto adverso del azucar y café comparado con el algodón sobre la esperanza de vida y la salud ya ha sido señalado en otras investigaciones 15.

\section{LOS DETERMINANTES DE LA NATALIDAD}

El Cuadro B presenta dos modelos para explicar la natalidad. En el primero (cuarta columna) se denota la significativa diferencia entre el comportamiento reproductivo en las plantaciones de algodón con respecto al azúcar y el café. Por otra parte, el desequilibrio entre los sexos afecta adversamente a la natalidad.

Las contribuciones independientes de las tasas de natalidad y mortalidad al crecimiento poblacional son dificiles de estimar. Es posible que las diferencias en tasas de natalidad entre plantaciones sean sólo el reflejo de diferentes tasas de mortalidad infantil, en la medida en que sólo se registrasen niños nacidos vivos. Debido a que no se posee una estimación de la mortalidad infantil para cada plantación durante el periodo, se estimó un segundo modelo -quinta columna del Cuadro B- que incluye la tasa de mortalidad de la plantación como control para eliminar el posible efecto de la diferente mortalidad infantil. El supuesto es que la tasa de mortalidad glohal puede considerarse un indicador indirecto de la tasa de mortalidad infantil, lo que no es necesariamente válido. Los resultados muestran que incluso cuando se controla por mortalidad, la tasa de natalidad de las plantaciones de algodón es significativamente más elevada que para el resto de las plantaciones.

La baja natalidad en los cultivos de azúcar y café pudo deberse a un control de la mujer de su fertilidad a través de la prolongada lactancia o abstinencia sexual. Sin embargo, ya que se está en presencia de una única región, con similares pautas culturales, la causa de distintos hábitos de lactancia o abstinencia sexual radicaría tanto en el efecto del cultivo como de la cultura recibida. Que las esclavas controlaban el número de hijos se denota a través del espaciamiento entre nacimientos, puesta en evidencia en varios estudios ${ }^{16}$. Convie-

15 Sobre el Caribe britanico en general, vease Higman (1984), p. 357; sobre Granada, Higman (1984), p. 361; sobre Surinam, Lamuer (1987), p. 322; sobre Trinidad, John (1988), p. 143.

16 Sobre Jamaica, vease Craton (1978), p. 96, y Dunn (1977), p. 55; una comparación de Virginia y Jamaica se presenta en Dunn (1977), p. 58; sobre Surinam, Lamuer (1987), p. 334; sobre el Caribe en general, Higman (1984), p. 357. 
ne examinar primero el efecto del cultivo sobre el trabajo femenino. La principal característica del trabajo de la mujer en azúcar en el Caribe es que requeria una continua labor en el campo a lo largo del año, al menos de unas diez horas diarias, sin interrupciones estacionales. Ello ocurria dado que cuando no se cosechaba la caña, los esclavos y esclavas se dedicaban a trabajar la tierra con azadas, pues los arados no se adaptaban al terreno pedregoso. Luego debian plantar la caña y cubrir los agujeros con tierra. Cuando las cañas crecian exigian una continua eliminación de malas hierbas. Finalmente se terminaba cosechando distintas siembras de caña, una labor que podia ocupar hasta seis meses, con un intenso ritmo de trabajo de unas 15 horas diarias, que incluian trabajo nocturno ${ }^{17}$. El que una mujer diera a luz no la eximia del trabajo: pocas semanas después de nacer el niño debía retornar a su cuadrilla. Si no deseaba dejar el niño bajo el cuidado de una esclava anciana debia llevarlo atado a la espalda, depositándolo en el suelo durante su trabajo ${ }^{18}$. Las condiciones para la mujer en el algodón eran menos agobiantes, por limitarse el trabajo de campo al tiempo de cosecha-que no exigia trabajo nocturno- y ser menor la cantidad de horas de labor requeridas. La cosecha duraba dos a tres meses, de los que unos 20 dias se interrumpian por lluvia ${ }^{19}$. Por otra parte, la utilización del arado, conjuntamente con el hecho de que la planta durara seis años, reducia mucho la preparación de la tierra, labor tan ardua en el azúcar. Fuera del tiempo de cosecha la mujer se dedicaba a separar y clasificar el algodón, a hilar y tejer, todas tareas que permitian tener a los niños con ellas ${ }^{20}$. El café estaria en una situación intermedia entre el azucar y el algodón. Aunque el tiempo de cosecha era largo, pudiendo durar tanto como el azúcar, unos seis meses, el resto del año era de baja demanda laboral. Además, las plantaciones de café estaban a mayor altura sobre el nivel del mar que las de azúcar; esto hacia que el clima más frio y las lluvias impidieran el trabajo continuo. Por otra parte, no existía la necesidad de un trabajo continuo de la tierra ${ }^{21}$. Las distintas exigencias de esfuerzo y tiempo de la mujer esclava en los diversos cultivos seria uno de los determinantes de la natalidad. El elevado coste de maternidad en plantaciones de azúcar, tanto por el impacto de trabajo adicional como por escasas posibi-

1: Sobre la continua e intensiva demanda de trabajo esclavo en el cultivo de azúcar, véase Craton (1978), p. 278; Higman (1984), pp. 162-166, 183-184; Dunn (1972), pp. 192, 200; Tomich (1990), pp. 140.150, 227.237.

18 Bush (1990), p. 121.

14 Earle (1992).

20 Véase Jones (1982), pp. 240, 252; Fox-Genovese (1988), p. 186.

21 Trouillot (1993), pp. 131-137; Geggus (1993), p. 93; Sheridan (1985), p. 242. 
lidades de atender al niño en horas de trabajo, fue un factor que hizo que la mujer buscara evitar la natalidad 22.

\section{CONCLUSIONES}

En este trabajo se ha enfatizado el impacto de los cultivos sobre el cambio demográfico. Las causas de los diversos regímenes demográficos no sólo parecen estar relacionadas con pautas culturales, posibilidades alimenticias o desequilibrios sexuales, sino también con el tipo de cultivo. En la muestra de plantaciones de Guyana se encontró para el algodón una tasa relativamente baja de mortalidad, con una tasa relativamente alta de natalidad. El café, por su parte, presentaba una baja natalidad; el azúcar, una baja natalidad y una alta mortalidad. Los resultados sugieren que en el Caribe y en Brasil el azúcar y en menor medida el café fueron responsables importantes de la caida de la población ${ }^{23}$. Los esclavos de Estados Unidos, predominantemente ocupados en el algodón, experimentaron sus efectos beneficiosos sobre el crecimiento natural de la po. blación.

El efecto de los cultivos sobre la natalidad ayudan a entender por qué una vez lograda la emancipación, ésta afectó mucho el ritmo de crecimiento natural en el Caribe ${ }^{24}$ y poco en Estados Unidos. Dado el impacto de las variables aqui presentadas, la explicación es directa: la emancipación redujo mucho más el coste de la maternidad en el Caribe que en Estados Unidos. En el Caribe la población comenzó a crecer por vez primera de manera notable - pese a que em. peoraron las condiciones sanitarias ${ }^{25}$ - al retirarse la mujer del mercado laboral y dedicarse plenamente a la maternidad ${ }^{26}$. Aunque el fenómeno laboral también ocurrió en Estados Unidos, no tuvo el mismo efecto sobre la natalidad ${ }^{27}$.

22 También en Trinidad la natalidad en azucar era menor que en los otros cultivos. Véase John (1988), p. 143.

${ }^{23}$ En el conjunto del Caribe Británico los esclavos trabajando en plantaciones de azuicar eran en 1810 el $56,8 \%$ del total y en 1830 el $62,7 \%$. En cafe y algodón estaban trabajando en 181012,3 o y $5,4 \%$, mientras que en 1830 las proporciones eran $9,4 \%$ y $1,7 \%$. (Higman (1984), p. 71). En Jamaica a fines del siglo ximu la proporcion de esclavos en azucar era 750 (Craton (1975), p. 254), aunque para 1832 la proporción habria bajado alli al $50 \%$, con un $14 \%$ dedica. do a café (Higman 1984, p. 54). En Barbados en 1770 la proporción en azucar era de $93 \%$ (CraIon (1975), p. 254); hacia 1834 se redujo al $78 \%$ (Higman (1984), p. 50). En Trinidad en 1810 la proporción era de 60,1 o (café 7,90 ) y en 1813 el 56,4 \% (café 6,8\%) (Higman (1984), p. 62). En Cuba en 1862 el $47 \%$ de los esclavos estaria en ingenios azucareros (Scott (1985). p. 12).

24 Craton (1978), p. 97.

25 Bush (1990), p. 136.

26 Véase Adamson (1975), p. 461, y Scott (1985), pp. 242.243.

2i Jones (1982), pp. 258-259. 


\section{BIBLIOGRAFÍA}

ADAMsox, Alan (1975): "The Reconstruction of Plantation Labor after Emancipation: the Case of British Guyana», en Engerman and Genovese, pp. 457.473.

Bircial), Laird W. (1990): Cuban Rural Society in the Nineteenth Century. The Social and Economic History of Monoculture in Matanzas, Princeton.

BRRIIN, Ira, y PhILIP D. Mokgan, eds. (1993): Cultivation and Culture. Labor and the Shaping of Slave Life in the Americas, Londres.

Bush, Barbara (1990): Slave Women in Carribbean Society 1650-1838. Londres.

CRATon, Michael (1975): «lamaican Slavery», en Engerman y Genovese (1975), pp. 249-284.

- (1978): Searching for the Invisible Man. Slaves and Plantation Life in Jamaica, Cambridge.

DeAN, Warren (1976): Rio Claro. A Brazilian Plantation System, 1820-1920, Stanford.

Dunv, Richard S. (1972): Sugar and Slaves. The Rise of the Planter Class in the English West Indies 1624-1713, Chapel Hill.

(1977): «A Tale of two plantations: Slave Life at Mesopotamia in Jamaica and Mount Airy in Virginia, 1799 to 1828", The William and Mary Quarterly XXXIV:1 (enerol, pp. 32- 65.

_ (1993): «Sugar Production and Slave Women in Jamaica», en Ira Berlin and Philip D. Morgan (1993), pp. 49.72.

EAR1.L, Carville (1992): «The Price of Precocity: Technical Choice and Ecological Constraint in the Cotton South, 1840-1890m, Agricultural History 66:3 (Summer), 25-60.

ENGFRnan, Stanley (1976): «ome Economic and Demographic Comparisons of Slavery in the United States and the British West Indies», The Economic History Revieu; XXIX:2, pp. $258-275$.

Encifrman, Stanley, y Eccine D. Ginovist, eds. (1975): Race and Slavery in Western Hemisphere: Quantitative Studics, Stanford.

Fogel, Robert W., y SMANity Excierdax (1974): Time in the Cross. The Economics of Negro Slavern. Boston.

- (1979): "Recent findings in the Study of Demography and Family Structure», Sociology and Social Research, 63, pp. 566-589.

Fox-Ginovest, Elizabeth 1988): Within the Plantation Houschold: Black and White Women of the Old South, Londres.

Glicicis, David P. (1978): «The Slaves of British-Occupied Saint Domingue: an analysis of the workforces of 197 absentee plantations, 1796-1797", Caribbean Studies 18: 1 y 2 (abril-julio), pp. 5.41 .

(1993): «Sugar and Coffee Cultivation in Saint Domingue and the Shaping of the Slave Labor Forte», en Berlin y Morgan (1993), pp. 73-96.

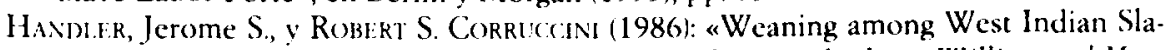
ves: Historical and Bioanthropological Evidente from Barbados», William and Mary Quarterly: XLIII:1, 111-117.

HiciNan, B. W. (1976): Slave Population and Economy in Jamaica 1807-1834, Londres.

- (1984): Slave Populations of the British Caribbean, Baltimore.

Jonn, A. Meredith (1988): The Plantation slaves of Trinidad 1793-1816. A mathematical and demographic inquin: Cambridge.

JoNFs, Jacqueline (1982): «My Mother was much of a woman: Black Women. Work and the Family Under Slavery", Feminist Siudies, 8:2 (Summer), pp. 235.269. 
KIPI.F, Kenneth (1984): The Caribbean Slave. A Biological History, Nueva York.

Ki.fin, Herbert S., y Stanley Encif.r.man (1978): «Fertility Differentials between Slaves in the United States and British West Indies: A Note on Lactation Practices and Their Possible Implications», William and Mary Quarterly, XXXV, 357-374.

LANU:R, Humphrey E. (1987): «Fertility differentials in three slave plantations in Suriname», Slavery and Abolition, 8:3 (diciembre), 313.335.

Merrick, Thomas W., y Douglas H. Graham (1979): Population and Economic Development in Brazil - 1800 to the present, Baltimore.

Roberts, G. W. (1977): «Movements in Slave Population of the Caribbean during the period of Slave Registration», en Vera Rubin y Arthur Tuden eds., Comparative Perspectives on Slavery in New World Plantation Societies, Nueva York, pp. 145-160.

Schw'ARTz, Stuart B. (1985): Sugar Plantations in the Formation of Brazilian Society - Babia, 1550-1835, Cambridge.

ScotT, Rebecca J. (1985): Slave Emancipation in Cuba. The Transition to Free Labor, Princeton.

ShFridan, Richard B. (1975): «Mortality and the Medical Treatment of Slaves in the British West Indies», en Engerman y Genovese (1975), pp. 249-284.

- (1985): Doctors and Slaves. A medical and demographic history of slavery in the British West Indies, 1680-1834, Cambridge.

Tomich, Dale W. (1990): Slavery in the Circuit of Sugar. Martinique and the World Economy, 1830-1848, Baltimore.

Troun.1.or, Michel-Rolph: «Coffee Planters and Coffee Slaves in the Antilles: The Impact of a Secondary Crop», en Berlin y Morgan (1993), pp. 124-137. 